\title{
Elephantiasis Nostras Verrucosa (ENV): A Complication of Congestive Heart Failure and Obesity
}

\author{
Drew Baird, MD, David Bode, MD, Troy Akers, DO, and Zachariah DeYoung, MD
}

Congestive heart failure (CHF) and obesity are common medical conditions that have many complications and an increasing incidence in the United States. Presented here is a case of a disfiguring skin condition that visually highlights the dermatologic consequences of poorly controlled CHF and obesity. This condition will probably become more common as CHF and obesity increase in the US. (J Am Board Fam Med 2010;23:413-417.)

Keywords: Elephantiasis, Congestive Heart Failure, Obesity, Lymphedema, Case Report

A 48-year-old man presented to our clinic complaining of 7 months of worsening, bilateral leg swelling with painful, oozing "water sores." He also described worsening dyspnea on exertion and 3-pillow orthopnea. His medical history was significant for poorly controlled congestive heart failure (CHF); obesity (body mass index, $43 \mathrm{~kg} / \mathrm{m}^{2}$ ); atrial fibrillation; and diabetes mellitus. He had no significant travel history or family history.

During physical examination vital signs showed mild hypoxia, tachypnea, and a fever of $101.6^{\circ} \mathrm{F}$. Jugular venous distension, bibasilar crackles, and an irregular heart rhythm were present. The lower legs revealed significant pitting edema with woody, indurated skin that had a circumferential confluence of weeping plaques with a pebbled, verrucous appearance (see Figures 1, 2, and 3).

Laboratory evaluation revealed normal complete blood count, cardiac enzymes, metabolic panel, and thyroid studies. Abnormal laboratory

This article was externally peer reviewed.

Submitted 2 June 2009; revised 9 October 2009; accepted 19 October 2009.

From the Department of Family and Community Medicine, Dwight D. Eisenhower Army Medical Center, Fort Gordon, GA.

Funding: none.

Conflict of interest: none declared.

Disclaimer: The opinions or assertions contained herein are the private views of the authors and are not to be construed as official or as reflecting the views of the Department of Defense.

Corresponding author: Drew Baird, MD, Department of Family and Community Medicine, Carl R. Darnall Army Medical Center, 36000 Darnall Loop, Fort Hood, TX 76544 (E-mail: drew.baird@us.army.mil).
Figure 1. Below both knees the patient had tense, woody edema with a circumferential confluence of white- to pink-colored plaques.

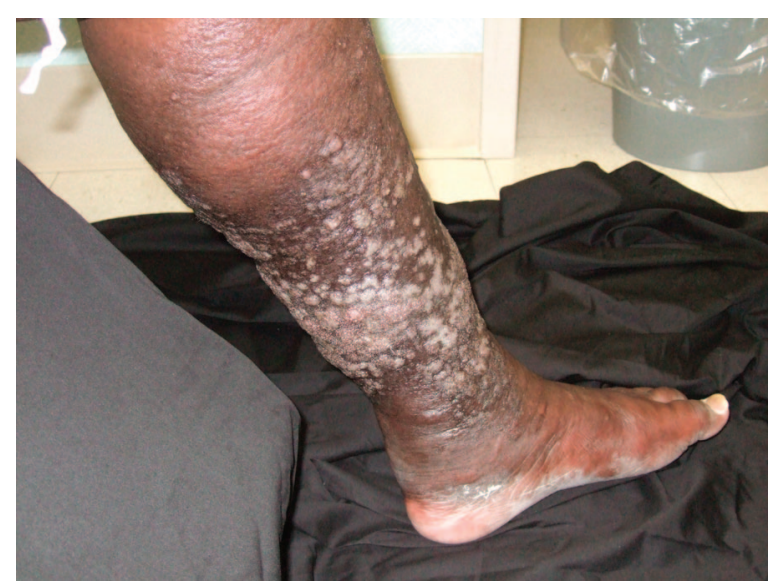

values included a C-reactive protein level of 4.1 $\mathrm{mg} / \mathrm{dL}$ (normal, 0 to 1.0 ) and brain naturetic peptide level of $342 \mathrm{pg} / \mathrm{mL}$ (normal, 0 to 100). Chest radiography showed diffuse pulmonary infiltrates and electrocardiogram showed atrial fibrillation. The patient was admitted for an acute CHF exacerbation and presumed cellulitis. A wound culture grew multiple organisms (Staphylococcus aureus, Serratia marcescens, Citrobacter koseri, Acinetobacter lwoffii) but blood and fungal cultures were negative. A skin punch biopsy was consistent with stasis dermatitis and, based on clinical examination, the patient was diagnosed with elephantiasis nostras verrucosa (ENV) with overlying acute lymphangitis. A conservative therapy was adopted to control his ENV, specifically antibiotics to treat the acute in- 
Figure 2. The patient's edema was seen especially from the mid-tibia to the ankle.

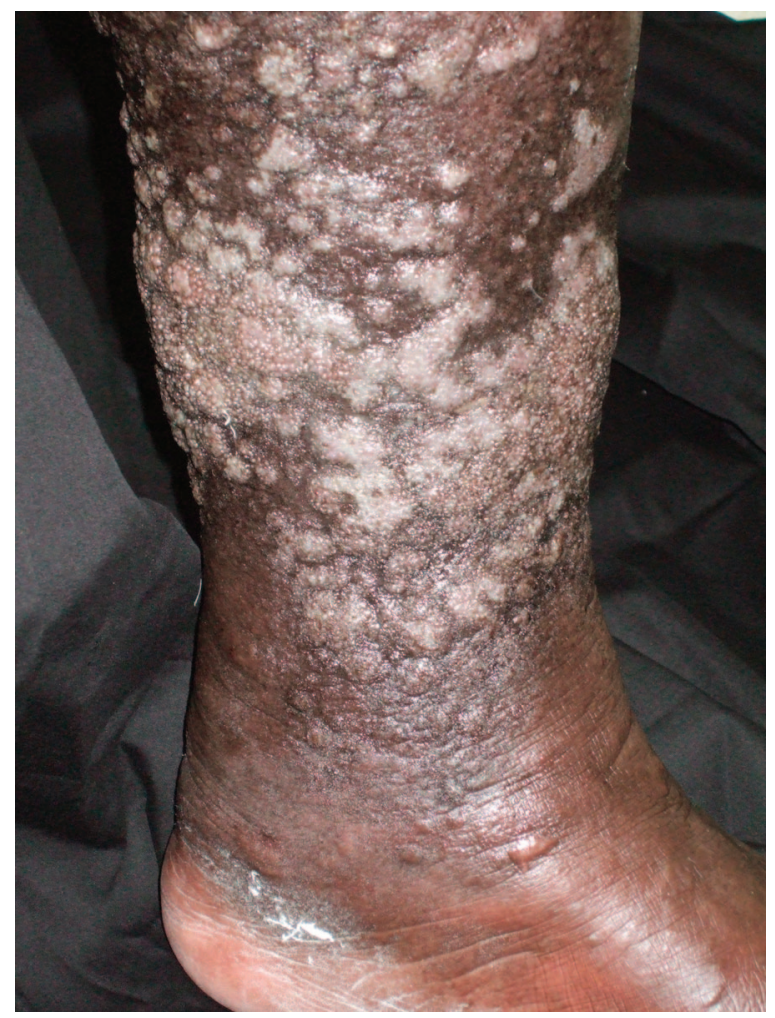

fection and leg elevation and compression stockings to manage the chronic ENV. Although discharged in stable condition, the patient died 3 months later from CHF-related complications.

\section{Discussion}

Elephantiasis nostras verrucosa (ENV) is the progressive disfiguring enlargement of a body part

Figure 3. The confluence of plaques had a verrucous, "cobblestoned" appearance with tightly packed, tense papules. Some papules had weeping ulcerations.

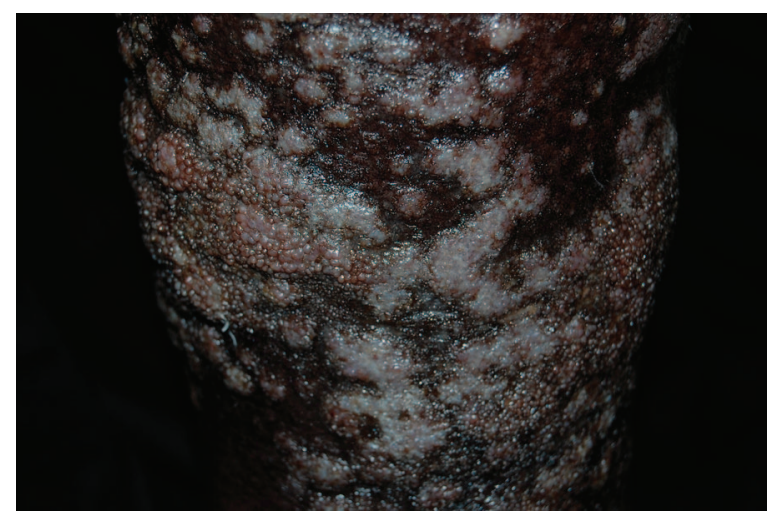

caused by recurrent soft tissue bacterial infections in the setting of chronic secondary lymphedema. In 1934 Castellani first used the word "nostras," meaning "from our region," ${ }^{1}$ to distinguish elephantiasis nostras from elephantiasis tropica, or filariasis, which is caused by the microscopic, parasitic Wuchereria worms that invade and obstruct the lymphatics. ENV typically appears in gravity-dependent regions, most commonly the lower extremities, but has been reported in the upper extremities, abdominal pannus, buttocks, orbital area, lips, ears, and scrotum. ${ }^{2-4}$

It is currently unclear which patients with chronic lymphedema progress to ENV. It is hypothesized that the protein-rich fluid that accumulates in the interstitium provokes an inflammatory response that impairs the local immune response and predisposes the patient to soft tissue infections such as erysipelas, cellulitis, and lymphangitis. Inoculation can result from insignificant trauma, poor hygiene, and dry fissured skin. ${ }^{1-7}$ With each bout of soft-tissue infection the lymphatics become increasingly fibrotic and the affected body part becomes more edematous and enlarged, even after the acute infection has subsided. Meanwhile, the skin undergoes epidermal hyperkeratosis and fibrosis of the dermis and subcutaneous tissues. ${ }^{2,4}$ In time this produces the characteristic clinical appearance of ENV: an enlarged body part with woody edema and thickened, hyperkeratotic skin that has a cobblestone-like, verrucous appearance. Over time, ulcerations become more common and become another route for infection.

$\mathrm{ENV}$ is a form of chronic secondary lymphedema; primary lymphedema is caused by congenital defects in the lymphatic system. ${ }^{5}$ Secondary lymphedema is the dysfunction of the lymphatic system caused by another primary disease process. Primary infectious etiologies include filarial, staphylococcal, and streptococcal infections, which cause direct inflammation and fibrosis of the lymphatic vessels. Noninfectious etiologies include disruption of the lymphatic system after trauma or surgery; obstruction by malignancy, CHF, and obesity; and lymphatic fibrosis by malignancy, radiation, venous stasis, CHF, obesity, portal hypertension, and scleroderma. ${ }^{2,5}$ The most common cause of secondary lymphedema in developing nations is filariasis; among industrialized nations it is malignancy. ${ }^{5}$ Two other causes of secondary lymphedema- 
CHF and obesity—are on the rise in the United States.

The prevalence of CHF is rising in America and it currently affects $2 \%$ of the US population. ${ }^{8}$ Approximately one quarter of the US population is obese and the prevalence of obesity is rising among all ages, all racial groups, all 50 states, and both sexes. ${ }^{9}$ As the prevalence of CHF and obesity rise, the complications of these diseases will also become more common; these complications include skin manifestations such as ENV.

$\mathrm{CHF}$ and its edema can be the beginning steps that lead to ENV. The decrease in myocardial contractility and cardiac output that is seen in CHF results in an increase in central venous pressure. This pressure is transmitted back to the capillary beds, where fluid leaks into the interstitium. The decreased cardiac output and capillary fluid leak create a functional decrease in intravascular volume, triggering various physiologic mechanisms to increase intravascular volume and thereby raising central venous pressure and worsening the edema. ${ }^{10}$ Beyond creating proteinaceous lymph, edema can directly overwhelm, impair, and obstruct the lymphatic system and create lymphedema.

Our patient's morbid obesity also played a role in the development of ENV. Obese patients already are at an increased risk for skin infections and impaired skin and soft tissue wound healing. With $\mathrm{ENV}$, it is theorized that excessive adipose tissue can impair lymphatic drainage and lead to the buildup of protein-rich lymphedema and associated fibrosis and inflammation. ${ }^{9}$ Our patient's CHF and obesity created "the perfect storm" to produce lymphedema, recurrent skin infections, and ultimately ENV.

ENV can be diagnosed clinically with history and physical examination alone, although other tools are available to assist the clinician. The physical examination finding of Stemmer sign-the inability to pinch the skin on the dorsal toes because of skin thickening - is suggestive of lymphedema. Although not necessary for ENV diagnosis, diagnostic studies can assist the clinician in evaluating lymphedema and ruling out other causes. Biopsy can rule out other causes of secondary lymphedema, such as malignancy. Histologically, ENV shows hyperkeratosis of the epidermis, loss of dermal papillae, fibrosis of the dermis and subcutaneous tissues, and widened lymphatic vessels. Lym- phoscintigraphy can assist in the diagnosis of lymphedema. Ultrasound can identify filarial adult worms within the lymphatics, although sensitivity is low. Computerized tomography and magnetic resonance imaging can be used to rule out obstructive malignancy. ${ }^{2,11}$ The differential diagnosis for ENV is broad and is listed in Table 1.

There currently is no established standard of care for the treatment of ENV. Therapies attempt to correct the underlying cause, maximize the patient's ability to use the affected limb, and prevent further complications. ${ }^{11}$ Initial conservative treatments include elevation of the affected limb, compression with bandages or stockings, massage, and pneumatic compression devices. ${ }^{1-3,11,13,14}$ During acute lymphangitis, appropriate antibiotics should be used to treat the infection. When there is no active infection, prophylactic use of antibiotics may be considered. ${ }^{3}$ There are several reports of success with the use of oral and topical retinoids, such as etretinate and tazarotene, respectively, which decrease epidermal proliferation, fibrogenesis, and inflammation. ${ }^{1-3}$ If medical measures fail, surgical options are available. These include debridement, lymphatic and lymphovenous anastomosis, lymphatic transplantation and, for severe cases in which the outlook of limb preservation is bleak, amputation. ${ }^{11,14}$

The prognosis for a patient with ENV is based on the severities of their ENV and their primary condition. Prognosis is improved when the disease process is interrupted early, the patient is motivated to participate in therapy, and there is close follow-up with a primary care physician. ${ }^{2,8,9}$ Depression, anxiety, and social isolation can develop because of embarrassing and grotesque features. Patients can suffer from disability caused by the loss of use of the affected limb. With further progression, bacterial and fungal super-infections of the skin and underlying bone can develop. The patient may ultimately die from sepsis associated with ENV or from the advancement of their primary condition. $^{2,7}$

\section{Conclusion}

Our patient had no identifiable malignancies, history of radiation therapy, surgical history, history of trauma, history of travel to tropical regions, or family history of similar pathology that would have caused his lymphedema. His advanced CHF and 


\begin{tabular}{|c|c|c|}
\hline Condition & Characteristics & References \\
\hline Primary (congenital) lymphedema & $\begin{array}{l}\text { Congenital abnormality in formation or function of } \\
\text { lymphatic vessels and has a morphology similar } \\
\text { to that of ENV. Onset is usually before the age } \\
\text { of } 25 \text { and there is often a family history of } \\
\text { lymphedema. }\end{array}$ & 5,6 \\
\hline Filariasis & $\begin{array}{l}\text { Caused by Wuchereria parasites blocking lymphatic } \\
\text { vessels and causing secondary bacterial } \\
\text { infections. Morphology similar to that of ENV. } \\
\text { Either endemic to tropical regions or a travel } \\
\text { history to these regions is present. Diagnosis is } \\
\text { confirmed on blood smear, antigen testing, or } \\
\text { identification of the parasite on tissue biopsy. }\end{array}$ & 2 \\
\hline Lipedema & $\begin{array}{l}\text { An abnormal amount of subcutaneous fat, typically } \\
\text { bilateral and symmetric, that spares the } \\
\text { feet."Stove pipe legs" with a negative Stemmer } \\
\text { sign. Early age of onset, usually female, with a } \\
\text { positive family history. Histologically has no } \\
\text { epidermal changes. }\end{array}$ & 2 \\
\hline Lipodermatosclerosis & $\begin{array}{l}\text { Caused by venous stasis and resulting in } \\
\text { subcutaneous fibrosis proximally that results in } \\
\text { an "inverted wine bottle" look to the affected } \\
\text { leg. }\end{array}$ & 2 \\
\hline Papillomatosis cutis carcinoides & $\begin{array}{l}\text { Morphology similar to that of ENV, typically } \\
\text { presents with ulcers, and is considered a low- } \\
\text { grade squamous cell carcinoma. Distinguished } \\
\text { from ENV by biopsy. }\end{array}$ & 1,2 \\
\hline $\begin{array}{l}\text { Pretibial myxedema (hyperthyroidism, Grave } \\
\text { disease) }\end{array}$ & $\begin{array}{l}\text { Caused by mucin deposition within the dermis, } \\
\text { causing nonpitting edema with thickened, } \\
\text { nodular plaques over anterior leg and dorsum of } \\
\text { the foot. Patients will have other sequelae of } \\
\text { hyperthyroidism (ie, exophthalmos) and will have } \\
\text { thyroid function test abnormalities. }\end{array}$ & 2,3 \\
\hline Stewart-Treves syndrome & $\begin{array}{l}\text { Angiosarcoma that develops in chronic } \\
\text { lymphedema and presents as an erythematous } \\
\text { mass. Seen in patients after radical mastectomy } \\
\text { and in congenital lymphedema. }\end{array}$ & 2,5 \\
\hline Venous stasis dermatitis & $\begin{array}{l}\text { Presents as a pitting edema with erythema, skin } \\
\text { desquamation, and pruritis. Does not have a } \\
\text { verrucous appearance. }\end{array}$ & 3 \\
\hline Deep venous thrombosis & $\begin{array}{l}\text { Acute onset of pitting edema, typically in lower } \\
\text { extremities, with no epidermal changes. }\end{array}$ & 5 \\
\hline Papular mucinosis & $\begin{array}{l}\text { Idiopathic disease distinguished by acid } \\
\text { glycosaminoglycan infiltration of the papillary } \\
\text { dermis. Presents clinically as waxy papules that } \\
\text { can coalesce into plaques. }\end{array}$ & 2 \\
\hline Podoconiosis & $\begin{array}{l}\text { Lymphatic obstruction caused by chronic, barefoot } \\
\text { walking on volcanic soils with high silica dust } \\
\text { content. }\end{array}$ & 3 \\
\hline
\end{tabular}

ENV, elephantiasis nostras verrucosa.

morbid obesity, however, contributed significantly to his lymphedema and resulting ENV. With an increasing prevalence of CHF and obesity in the United States, their complications will also increase in prevalence. ENV is, unfortunately, a disease without well-studied, standardized, or effective treatment, and its prognosis is poor. For the clinician, this case illustrates the importance of early intervention for patients with $\mathrm{CHF}$ and obesity to prevent sequelae such as ENV. Ultimately, the best therapy for $\mathrm{ENV}$, as with all complications of $\mathrm{CHF}$ and obesity, is primary prevention.

\section{References}

1. Schissel DJ, Hivnor C, Elston DM. Elephantiasis nostras verrucosa. Cutis 1998;62:77-80.

2. Sisto K, Khachemoune A. Elephantiasis nostras verrucosa: a review. Am J Clin Dermatol 2008;9:141-6. 
3. Boyd J, Sloan S, Meffert J. Elephantiasis nostrum verrucosa of the abdomen: clinical results with tazarotene. J Drugs Dermatol 2004;3:446-8.

4. Castellani A. Researches on elephantiasis nostras and elephantiasis tropica with regard to their initial stage of recurring lymphangitis (lymphangitis recurrens elephantogenica). J Trop Med Hyg 1969;72:89-96.

5. Tiwari A, Cheng K, Button M, Myint F, Hamilton G. Differential diagnosis, investigation, and current treatment of lower limb lymphedema. Arch Surg 2003;138:152.

6. Vaccaro M, Borgia F, Guarneri F, Cannavo S. Elephantiasis nostras verrucosa. Int J Dermatol 2000;39: 764.

7. Yoho RM, Budny AM, Pea AS. Elephantiasis nostras verrucosa. J Am Podiatr Med Assoc 2006;96:442-4.

8. Hunt SA, Abraham WT, Chin MH, et al. 2009 focused update incorporated into the ACC/AHA 2005 guidelines for the diagnosis and management of heart failure in adults: a report of the American College of Cardiology Foundation/American Heart Association Task Force on Practice Guidelines developed in collaboration with the International So- ciety for Heart and Lung Transplantation. J Am Coll Cardiol 2009;53:e1-e90.

9. Yosipovitch G, DeVore A, Aerlyn D. Obesity and the skin: skin physiology and skin manifestations of obesity. J Am Acad Dermatol 2007;56:901-16.

10. Navas JP, Martinez-Maldonado M. Pathophysiology of edema in congestive heart failure. Heart Dis Stroke 1993;2:325-9.

11. Turhan E, Ege A, Keser S, Bayar A. Elephantiasis nostras verrucosa complicated with chronic tibial osteomyelitis. Arch Orthop Trauma Surg 2007;128: 1183-6.

12. Duckworth AL, Husain J, Deheer P. Elephantiasis nostras verrucosa or "mossy foot lesions" in lymphedema praecox: report of a case. J Am Podiatr Med Assoc 2008;98:66-9.

13. Iwao F, Sato-Matsumura KC, Sawamura D, Shimizu H. Elephantiasis nostras verrucosa successfully treated by surgical debridement. Dermatol Surg 2004;30:939-41.

14. Motegi S, Tamura A, Okada E, Nagai Y, Ishikawa O. Successful treatment with lymphaticovenular anastomosis for secondary skin lesions of chronic lymphedema. Dermatology 2007;215:147-51. 